\title{
Lung transplantation for acute COVID-19: the Toronto Lung Transplant Program experience
}

\author{
Jonathan C. Yeung MD PhD, Marcelo Cypel MD, Cecilia Chaparro MD, Shaf Keshavjee MD
}

— Cite as: CMAJ 2021 September 27;193:E1494-7. doi: 10.1503/cmaj.211143

\section{Case 1}

A 60-year-old previously healthy man was admitted to hospital with COVID-19 pneumonia that was treated initially with noninvasive ventilation, steroids and antibiotics. Six weeks after admission, the patient remained dependent on oxygen, using a highflow nasal cannula combined with a nonrebreather mask. Physical examination found proximal muscle wasting due to the long hospital stay. Computed tomography (CT) of his chest showed bilateral dense consolidations with superimposed interstitial and fibrotic changes. Because we thought the fibrosis was unlikely to resolve, we discussed the option of lung transplantation with him and his family, both of whom were interested in the procedure. An acute clinical deterioration subsequently led to his intubation, transfer to our extracorporeal life support (ECLS) centre and placement on veno-venous extracorporeal membrane oxygenation (V-V ECMO) as a bridge to transplantation. Seventeen days after ECMO cannulation, the patient underwent successful double lung transplantation with removal of the V-V ECMO immediately after transplant. On postoperative day 37 , he no longer needed oxygen and we discharged him to a rehabilitation centre. At follow-up 5 months later, he was at home and did not require oxygen.

\section{Case 2}

A 53-year-old previously healthy man was admitted to hospital with COVID-19 pneumonia and received treatment with highflow nasal cannula oxygen, steroids and antibiotics. The patient was admitted to the intensive care unit (ICU) for 2 weeks but avoided intubation, improved clinically and was discharged to the ward on high-flow oxygen. Two weeks later, his condition deteriorated and CT showed extensive ground glass opacities, fibrosis and traction bronchiectasis in his lungs. We administered additional antibiotics, and he was intubated, paralyzed and proned. He was transferred to our ECLS centre and placed on V-V ECMO. Under ECMO support, he was extubated and participated in bedside physiotherapy. After no improvement in lung function after 7 weeks on ECMO and discussion with the patient and his family, we listed him for lung transplantation. Twenty-five days after listing, and 60 days after the start of ECMO, we performed a successful double-lung transplantation with removal of $\mathrm{V}-\mathrm{V}$

\section{Key points}

- Lung transplantation for COVID-19 acute respiratory distress syndrome (ARDS) is feasible but requires careful assessment of candidacy.

- Single-organ failure, ability to consent, participation in physiotherapy and negative results for COVID-19 polymerase chain reaction tests are key considerations when evaluating candidacy for lung transplantation in patients with acute ARDS.

- Selected patients with ARDS-related pulmonary fibrosis after COVID-19 may be lung transplant candidates in the future.

ECMO immediately after transplant. We discharged the patient, no longer requiring oxygen, to our rehabilitation centre 25 days after the surgery. At follow-up 4 months later, he was at home and did not require oxygen.

\section{Case 3}

A 48-year-old man with a previous history of gout was admitted with severe $\alpha$ (B.1.1.7) variant COVID-19 pneumonia, intubated and, 5 days later, proned. The patient received treatment with steroids and antibiotics, and acquired severe acute kidney injury that required dialysis 6 days after intubation. His condition continued to deteriorate and he was transferred to our ECLS centre for V-V ECMO. He could not be extubated because of hypoxia and subsequently underwent tracheostomy. Five weeks after cannulation for ECMO, spontaneous rupture of 2 right lower branches of the pulmonary artery led to a large hemothorax, which we treated with massive transfusion and coiling of the artery branches. We drained the hemothorax by tube thoracostomy and administered tissue plasminogen activator to liquify organized clot and later converted to an empyema tube. Complications included several infections, delirium and deconditioning over the next 6 weeks; however, the patient's kidney injury ultimately recovered and he was weaned off dialysis. Given the substantial destruction of his lungs, we did not expect recovery. Given the empyema from the infected hemothorax, the patient's long duration of delirium, ICU-related sarcopenia and possible limited understanding of lung transplantation, we were 


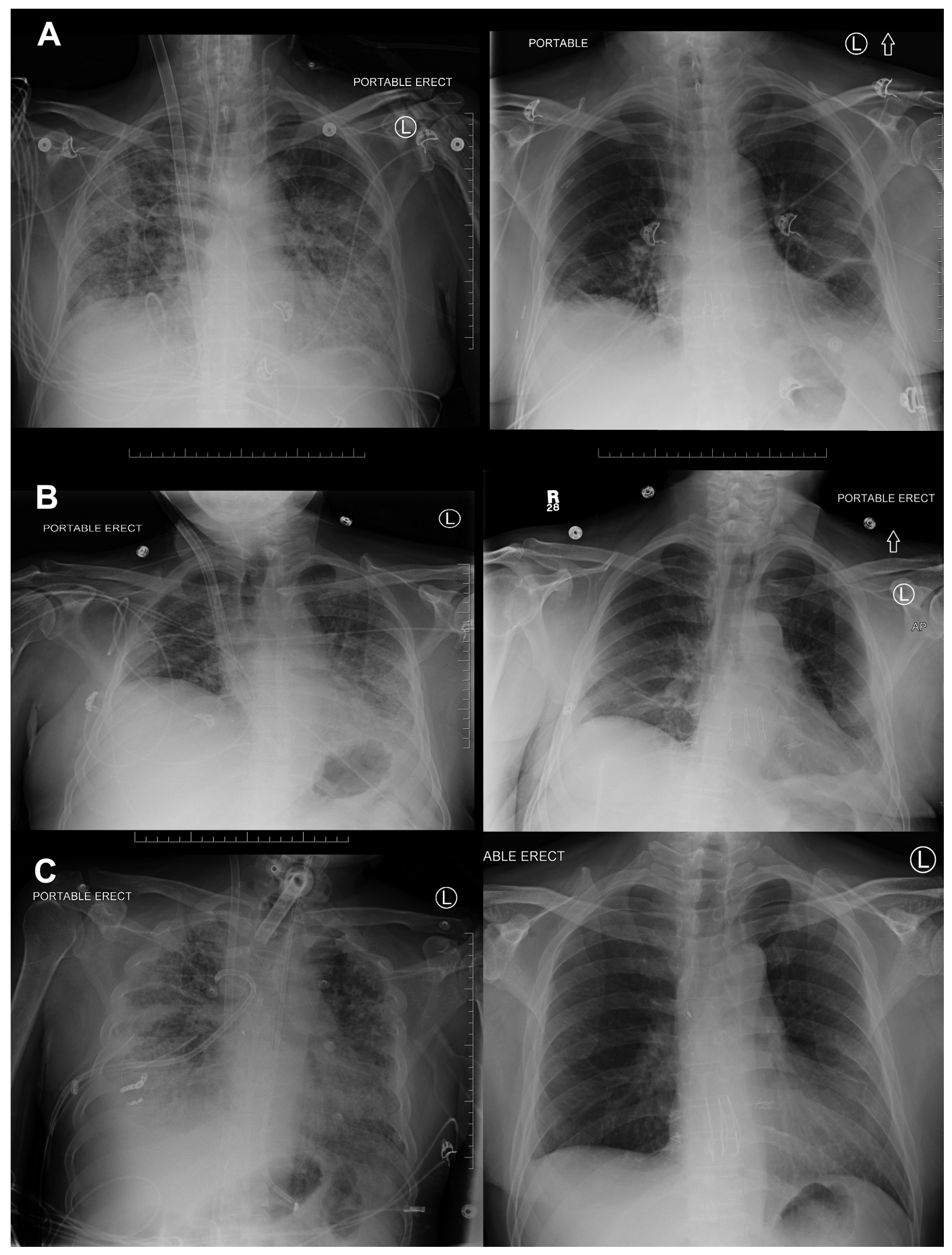

Figure 1: Chest radiographs before and after lung transplantation for (A) Case 1, (B) Case 2 and (C) Case 3. 
concerned about his suitability for lung transplant. However, 75 days after ECMO cannulation, his delirium improved and he was able to respond to commands and participate in physiotherapy. He did not have the capacity to consent because of continued ventilation and sedation. However, his wife indicated that she was confident that he would wish to undergo lung transplantation. About 3 months after ECMO cannulation, we placed the patient on the transplant list and performed a successful doublelung transplantation 2 days after this listing. We removed V-V ECMO immediately after the transplantation and discharged the patient to rehabilitation on postoperative day 22, with no requirement for oxygen. At follow-up 4 months later, the patient remained at home and did not need oxygen.

Chest radiographs before and after lung transplantation (before discharge) for the 3 cases are shown in Figure 1. Pathology reports from the lungs that were removed from all 3 patients showed diffuse alveolar damage consistent with COVID-19 infection. Table 1 provides the results of pulmonary function tests for these patients at 3 months after lung transplantation, which showed good clinical function and spirometry values as expected following a double lung transplant. ${ }^{1}$

\section{Discussion}

The COVID-19 pandemic has resulted in a substantial number of patients progressing to respiratory failure in Canada. A subset of patients have such profound lung injury from COVID-19 acute respiratory distress syndrome (ARDS) that conventional ventilation is inadequate and ECLS is used to bridge these patients to recovery. ${ }^{2}$ Although some patients ultimately recover, some patients become completely dependent on ECLS for survival for months, and imaging usually shows pulmonary fibrosis and bronchiectasis that is unlikely to improve. At this point, lung transplantation becomes the only option, but the ideal timing of transplantation in these patients is still not known because a balance must be achieved between doing it "too early" (when lung injury may yet reverse) versus "too late" (when the patient is unlikely to recover and the procedure is futile).

Although death is one definition of futility, transplantation in a patient who has no desire to live with a lung transplant or who is unable to recover to a desirable state of overall functioning is also futile. Lung transplantation requires patients and their caregivers to commit to a long period of recovery after surgery, lifelong immunosuppression and the ramifications thereof, and overall median long-term survival is only around 6 years. ${ }^{3}$ Conventional assessment for candidacy for lung transplantation is comprehensive; the expectations and social supports available to a candidate are as important as medical factors. Transplant assessment in the acute setting of ECLS is more challenging, in part because physicians lack experience with the natural history of COVID-19. Decision-making in this context requires consideration of the acuteness of the functional decline, possible inability to fully assess the wishes of the patient, uncertainty of family support after the operation and unclear physical recovery.

To guide assessment of COVID-19 for lung transplantation, we evaluate both disease and social considerations to maximize suc-

\begin{tabular}{|c|c|c|c|c|c|}
\hline Patient & $\begin{array}{l}\text { FEV1, } \\
\text { L (\%) }\end{array}$ & $\begin{array}{l}\text { FVC, } \\
\text { L (\%) }\end{array}$ & $\begin{array}{c}\text { FEV1/FVC, } \\
(\%)\end{array}$ & $\begin{array}{l}\text { 6MWT, } \\
\text { m (\%) }\end{array}$ & $\begin{array}{c}\text { Oxygen } \\
\text { requirement } \\
\text { on exertion }\end{array}$ \\
\hline 1 & $1.9(50)$ & $2.5(49)$ & 75 (99) & 207 (33) & RA \\
\hline 2 & $1.6(38)$ & $2.6(46)$ & $61(78)$ & $240(37)$ & RA \\
\hline 3 & $2.1(58)$ & $2.6(55)$ & $80(100)$ & $357(60)$ & RA \\
\hline
\end{tabular}

Note: FEV1 = forced expiratory volume in 1 second, FVC = forced vital capacity, RA = room air, 6MWT = 6-minute walk test.

cess. At present, we aim for an age younger than 65 years, isolated lung dysfunction, radiologic evidence of irreversible lung disease such as fibrosis and a current negative test result for COVID-19 polymerase chain reaction (PCR). ${ }^{4}$ The negative PCR result reduces the likelihood of ongoing active infection at the time of transplantation and reinfection of the graft. ${ }^{5}$ Our 3 patients met all of these criteria. We also aim to discuss transplantation with the patient directly to confirm that a lifestyle after lung transplant is desirable and emphasize that they should be motivated enough to participate in physiotherapy to maintain strength for postoperative recovery. Our criteria are similar to those proposed by other centres in Canada and in other countries with higher incidence of COVID-19. ${ }^{6}$

Our first patient made an initial recovery from his acute SARSCoV-2 infection and subsequently deteriorated from fibrotic changes after acquiring ARDS. Therefore, the transplant team had the opportunity to discuss transplantation in detail before starting ECMO as a bridge to transplant. The second patient's condition was more acute in that he was placed on ECMO as a bridge to recovery. However, when irreversible damage to his lungs was determined, we were able to discuss lung transplantation in the acute setting with him. The third patient was most challenging because we could not have a direct discussion with him owing to his delirium. Although it resolved just before his transplant, it was unclear if he could fully understand the complexities of lung transplantation so soon after a long and profound delirium. Most of our discussion was with his wife who, we thought, after lengthy discussions, had his best interests in mind. Consent by substitute decision-makers increases the risk of future regret in transplant recipients because of the considerable lifestyle changes and complications that can occur after transplantation, although this patient has been extremely happy with his post-transplant course.

The major risk to long-term quality of life in patients who undergo lung transplant following COVID-19 pneumonia is the long rehabilitation period that is needed after the transplant procedure. The ICU-acquired weakness from prolonged ventilation and ECMO support, often with long periods of paralysis, can also affect recovery. ${ }^{7}$ Rehabilitation is commonly required after lung transplantation and, once acute illness is managed, patients are transferred to a rehabilitation hospital familiar with the needs of transplant recipients. Close ties between the rehabilitation facility and the transplant team allows for timely transfer back to the transplant centre if acute complications develop. Likely because our patients were in good 
health before acquiring COVID-19, we saw a remarkable and faster recovery of physical capacity after transplantation than other cohorts of patients who have a worse baseline health status.

As our patients were on ECMO during their time on the transplant list, they had the highest priority. Donor lungs are scarce, and a balance between the needs of this very sick group of patients and others waiting on the list needs to be reached. We performed ongoing reassessment of transplant volumes and waitlist mortality during the care of these patients and were reassured that lung transplantation in these patients was a reasonable course of action. Over 3 waves of COVID-19, at the time of writing, 151 patients have been placed on ECMO in our centre and only the 3 patients presented received transplants. During that time, 149 other patients received lung transplants for diseases unrelated to COVID-19. Indications for lung transplantation will evolve with time, but transplantation for COVID-19 on ECMO does not appear to threaten patients currently on the waitlist.

With increased vaccination rates among Canadians, we expect that the incidence of COVID-19 requiring ECMO will decrease substantially in the future. We currently have fewer than 5 patients with acute COVID-19 on ECMO who are awaiting transplant. As more patients recover from COVID-19, more with long-term sequelae of SARS-CoV-2 infection, such as fibrosis, may require lung transplantation. ${ }^{8}$ Indeed, we currently have fewer than 5 patients on the waiting list for end-stage fibrotic lung disease following COVID-19 who survived but require oxygen.

Over time, more worldwide experience will improve decisionmaking on lung transplantation for acute COVID-19. Our experience to date aligns with other published case reports and series. ${ }^{6,9,10}$ A 2021 case series reported on 12 patients with COVID19 who were supported by ECMO in 4 countries and underwent lung transplantation after considerations of criteria that aligned with ours (i.e., age $<65 \mathrm{yr}$, single organ failure, adequate social supports and ability to participate in decision-making where possible). ${ }^{6}$ In 2020, a case series of 2 patients in China, both older than 65 years of age, in whom CT of the lungs showed consolidation but no obvious fibrosis, reported good early outcomes on postoperative days 2 and $5 .{ }^{9}$ We would likely not have offered transplantation to these 2 reported cases because of the older age and lack of "end-stage" manifestations of lung disease at the time of transplantation. Another 2020 report described a patient in Austria with positive PCR test results for SARS-CoV-2 but a negative result for culture who underwent transplantation with a good short-term outcome. ${ }^{10}$

\section{Conclusion}

Our 3 cases with good short-term outcomes show the feasibility and considerations of lung transplantation in patients following severe acute COVID-19 in Toronto. Both survival and quality of life are important to determine the long-term benefit of lung transplantation for COVID-19 ARDS, and international experience and collaboration over time will enhance learning. We anticipate that lung transplantation will be considered an attractive treatment option for selected patients with acute or chronic fibrotic ARDS due to COVID-19.

\section{References}

1. Mason DP, Rajeswaran J, Li L, et al. Effect of changes in postoperative spirometry on survival after lung transplantation. J Thorac Cardiovasc Surg 2012; 144:197-203.

2. Barbaro RP, MacLaren G, Boonstra PS, et al.; Extracorporeal Life Support Organization. Extracorporeal membrane oxygenation support in COVID-19: an international cohort study of the Extracorporeal Life Support Organization registry. Lancet 2020;396:1071-8.

3. Yusen RD, Edwards LB, Dipchand AI, et al.; International Society for Heart and Lung Transplantation. The Registry of the International Society for Heart and Lung Transplantation: thirty-third Adult Lung and Heart-Lung Transplant report - 2016; Focus theme: primary diagnostic indications for transplant. $J$ Heart Lung Transplant 2016;35:1170-84.

4. Cypel M, Keshavjee S. When to consider lung transplantation for COVID-19. Lancet Respir Med 2020;8:944-6.

5. Cevik M, Tate M, Lloyd O, et al. SARS-CoV-2, SARS-CoV, and MERS-CoV viral load dynamics, duration of viral shedding, and infectiousness: a systematic review and meta-analysis. Lancet Microbe 2021;2:e13-22.

6. Bharat A, Machuca TN, Querrey M, et al. Early outcomes after lung transplantation for severe COVID-19: a series of the first consecutive cases from four countries. Lancet Respir Med 2021;9:487-97.

7. Lad H, Saumur TM, Herridge MS, et al. Intensive care unit-acquired weakness: not just another muscle atrophying condition. Int J Mol Sci 2020;21:7840.

8. Spagnolo P, Balestro E, Aliberti S, et al. Pulmonary fibrosis secondary to COVID-19: A call to arms? Lancet Respir Med 2020;8:750-2.

9. Han W, Zhu M, Chen J, et al. Lung transplantation for elderly patients with endstage COVID-19 pneumonia. Ann Surg 2020;272:e33-4.

10. Lang C, Jaksch P, Hoda MA, et al. Lung transplantation for COVID-19-associated acute respiratory distress syndrome in a PCR-positive patient. Lancet Respir Med 2020;8:1057-60.

\section{Competing interests: None declared.}

This article has been peer reviewed.

The authors have obtained patient consent.

Affiliation: Toronto Lung Transplant Program, Toronto General Hospital, Toronto, Ont.

Contributors: All of the authors wrote and revised the manuscript, gave final approval of the version to be published and agreed to be accountable for all aspects of the work.

Content licence: This is an Open Access article distributed in accordance with the terms of the Creative Commons Attribution (CC BY-NCND 4.0) licence, which permits use, distribution and reproduction in any medium, provided that the original publication is properly cited, the use is noncommercial (i.e., research or educational use), and no modifications or adaptations are made. See: https://creativecommons.org/ licenses/by-nc-nd/4.0/

Correspondence to: Marcelo Cypel, marcelo.cypel@uhn.ca

The section Cases presents brief case reports that convey clear, practical lessons. Preference is given to common presentations of important rare conditions, and important unusual presentations of common problems. Articles start with a case presentation ( 500 words maximum), and a discussion of the underlying condition follows (1000 words maximum). Visual elements (e.g., tables of the differential diagnosis, clinical features or diagnostic approach) are encouraged. Consent from patients for publication of their story is a necessity. See information for authors at www.cmaj.ca. 